\title{
ECLIPSING BINARIES IN MULTIPLE STAR SYSTEMS
}

\author{
C. R. CHAMBLISS \\ Kutztown University, Kutztown, Pennsylvania, USA 19530
}

Although the majority of the known eclipsing binaries are in systems which consist of only the two eclipsing components, a significant number of them do occur as components of triple star systems or higher order multiple star systems. In this context this term is restricted to those systems whose components are in closed orbits. A recent survey of visual binaries with variable components is that by Proust et al. (1981), which detalls about 300 systems. Walker (1979) has also prepared a list of variables as components of double star systems, and this includes numerous systems not included in other lists.

These catalogues surveyed all types of variable stars or suspected variables which are observed in visual double star systems. A different approach to this topic was taken by Fekel (1981), who examined the properties of close multiple stars. Both eclipsing and non-eclipsing spectroscopic binaries were considered, but the upper limit of the period for the third component in each system was restricted to about 200 years. Fekel's survey examined 43 systems, of which six have eclipsing binaries as components.

In this survey the properties of about 80 multiple star systems which contain eclipsing binaries as components are reviewed. Most of these are triple star systems in which the third component is visually resolved from the eclipsing pair. But in several cases the third component is detectable only by spectroscopy or with such techniques as speckle interferometry.

What constitutes verification that a given eclipsing binary is indeed a member of a multiple star system? If the eclipsing binary is a component of a fairly close visual binary system and if the components of that system display orbital motion or common proper motions, then one can be quite certain that the system is indeed multiple. But many of the systems listed in the catalogue of Proust have widely-spaced visual components. I have generally limited the survey of systems with visual components to those with $\rho=20$ " or less. If the third component is too close to be resolved visually, it may be resolved by speckle interferometry or by some other interferometric technique.

It is also possible that the third component will leave a spectral signature in the spectrum of the eclipsing binary. The spectra of both Algol and V505 Sgr display sharp lines which arise from the third components of these systems, and both of these systems are now also resolved by speckle interferometry. It is also possible that the third component will cause a variable $\gamma$-velocity in the system of the eclipsing pair. VV Orionis has long been known to have a third component, since its $\gamma$-velocity is variable despite the fact that the third component itself is much too faint to display distinct lines in the spectrum of the eclipsing pair. 
A third component will orbit about the eclipsing pair, and this mutual motion will produce a light-time effect in the O-C residuals of the times of minimum light of the eclipsing pair unless the orbit of the third component is almost exactly in the plane of the sky. A good example is the well-known term with a semi-amplitude of nine minutes which arises from the orbital effect which Algol $\mathrm{C}$ has on the times of minimum light of this eclipsing pair. Residuals in the times of minimum light of eclipsing binaries can arise from many other causes, however, and one must not immediately jump to the conclusion that all eclipsing binaries which display sinousoidal $\mathrm{O}-\mathrm{C}$ residuals in their times of minimum light necessarily have third components.

In the early days of the analysis of eclipsing binary light curves only the spherical model was generally available, and often this did not give a very good approximation to the geometry of many eclipsing binaries. Investigators sometimes found that they could improve matters by adding a "third light" to the system. Such a procedure is valid only if there is clear evidence that a third component actually exists in a given system. In more recent years the use of more sophisticated models for dealing with the light variations of eclipsing binaries have made it unnecessary to add such a correction except in those cases where a third light is definitely present.

Although triple star systems are by far the most common of the multiple star systems which contain eclipsing binaries, higher-order systems do exist. Quadruple systems tend to be either of hierarchy 2 (two pairs of close double stars widely spaced) or of hierarchy 3 (four stars involving three quite different spacings and orbital periods). An example of the former is the quadruple system which contains the eclipsing binaries BV and BW Draconis. Eta Orionis is an excellent example of the latter type of quadruple system.

Quintuple and sextuple systems are much rarer, but the system HR 3337. which contains an eclipsing binary with a period of $2^{\mathrm{d}} .50$, appears to be quintuple. Castor is a well-known sextuple system, which contains the eclipsing binary YY Geminorum. Sextuple stars are extremely rare, however, and the only other well-known case is Beta Tucanae, a system which contains no eclipsing binaries.

Trapezium-type systems are often considered as multiple star systems, but they can also be regarded as compact star clusters which contain numerous stars separated by comparable distances. The Trapezium itself is the center of an extensive cluster in Orion which contains two eclipsing binaries, BM Orionis ( $\theta^{\prime}$ Ori B) and V1016 Orionis ( $\theta^{\prime}$ Ori A). Another example of an eclipsing binary in a star cluster is SZ Cam which together with its visual companion, ADS 2984 A, are the brightest stars in the compact cluster NGC 1502.

In most triple star systems the orbit of the third component is not co-planar with that of the close pair. In some instances the value of $\phi$, the minimum inclination between these orbital planes, is less than $10^{\circ}$. Examples include $\lambda$ Tauri and V772 Herculis, but normally even very close triple systems have 
orbits which are not co-planar. Although the eclipsing binaries in multiple star systems usually have circular orbits, this is almost never the case for those of the third component. The most extreme case is that of V772 Herculis, where the eccentricity of the orbit of the third component is 0.958 .

The ratios of period $\left(\mathrm{P}_{2} / \mathrm{P}_{1}\right)$ and of semimajor axes $\left(\mathrm{a}_{2} / \mathrm{a}_{1}\right)$ in the triple star systems vary over a huge range. Three of the systems in this survey $(\lambda$ Tauri, $V V$ Orionis, and IU Aurigae) have values of $\mathrm{P}_{2} / \mathrm{P}_{1}$ of only $8.35,80.8$, and 162 . respectively. These systems all involve early $B$-type main sequence stars as components of the eclipsing pairs, and this type of system is the one most frequently encountered in multiple star systems, especially close ones. In IU Aurigae, the third component produces a strong perturbation in the orbit of the eclipsing pair which results in the inclination of the latter varying over a wide range in a period of several decades. In $\lambda$ Tauri this effect is also pronounced, but is is not as great as is that of IU Aurigae since the third component of $\lambda$ Tauri is far less massive than is the eclipsing pair of this system.

Far more frequently encountered are systems in which the value of $P_{2} / P_{1}$ is many thousands. In the quadruple system BV Dra $\left(P=0^{d} .3501\right)$ and BW Dra $\left(P=0^{d} .2922\right)$, the two pairs can be estimated to be about $1200 \mathrm{AU}$ apart from each other, and the period of mutual revolution would be about 24,000 years. This value is about $3 \times 10^{7}$ times the mean value of the orbital periods of the two binary pairs.

In systems with four or more components, systems of hierarchy 3 are sometimes encountered. A good example is Eta Orionis. In this system there is an eclipsing pair with a period of $7^{d} .984$. Eta Ori $A$ also contains a third component, resolvable with speckle interferometry, which has an orbital period of about 9 years. Eta Ori B is at $\rho=1 " .60$ and has a period of about 2300 years about the components of Eta Ori $A$. In this system the ratio $P_{2} / P_{1}$ is about 400 . while that of $\mathrm{P}_{3} / \mathrm{P}_{2}$ is about 250 .

AK Herculis may be another quadruple system of this type, but that is far from certain. The eclipsing pair has $P=0^{d} .4215$, and there is a visual component at $\rho=4 " .7$, which would imply an orbital period of a few millennia. But AK Her exhibits an apparent light-time effect whose period is estimated to be in the range 58 to 78 years. This would imply a separation of about $24 \mathrm{AU}$ or $\rho=0 " .25$. Such a component should be resolvable, but no companion at about this distance has been seen.

Castor is the only known sextuple system which includes an eclipsing binary. Castor A and Castor B are each single-lined spectroscopic binaries with periods of $9^{d} .213$ and $2^{d} .928$, respectively. Their mutual period of revolution is about 450 years. YY Gem, the eclipsing binary, is at $\rho=71 "$, implying a minimum separation of about $1000 \mathrm{AU}$ and a period of at least 15,000 years.

As has been noted, eclipsing binaries which contain early B-type stars are 
most frequently encountered in multiple star systems. Another group which often has additional components are the W UMa-type stars. In this survey 12 members of this group (GZ And, 44i Boo, VW Cep, AA Cet, CC Com, BV Dra, BW Dra, AK Her, AM Leo, HT Vir, AW UMa, and W UMa) all seem confirmed as members of multiple star systems. In addition to the three systems referred to previously (BV Dra, BW Dra, and AK Her), AA Cet is a component of a quadruple star system, and GZ And may even be in a quintuple system.

Two groups of interacting binaries are conspicuous by their rarity in multiple star systems. These are the RS CVn stars and the cataclysmic binaries. Among well-known examples of the former group only V711 Tauri has a visual companion, but the interacting pair is non-eclipsing. Among the latter group only CQ Dra, a close companion of the red giant $4 \mathrm{Dra}$, is known to be multiple. This variable, however, is also non-eclipsing. A former theory, now largely discredited, is that the W UMa-type stars might be the progenitors of the cataclysmic binaries. If this were the case, however, the discrepancy between the abundance of W UMa-type stars in multiple systems and the great rarity of cataclysmic binaries in such systems would have to be accounted for.

This survey finds that only about 80 of the well-known eclipsing binaries are in multiple star systems. This is less than $2 \%$ of the total known eclipsing binaries, but many of these are very poorly known, so this proportion will undoubtedly rise as new data are accumulated. Quadruple stars with eclipsing binaries are much less common. In addition to those already mentioned, NO Puppis certainly falls into this class. As has been noted, the Trapezium in Orion contains two eclipsing binaries, but it is basically a compact star cluster rather than a multiple star system, the components of which have closed orbits.

Although different researchers have come to varying conclusions as to the frequencies of multiple star systems, it is felt that about $20-30 \%$ of all binary stars should at least be triple, and of these $20-30 \%$ should be quadruple, etc. Among eclipsing binaries only about one system in 15 which is at least triple has four or more components. Perhaps this finding should be regarded as enigmatic. There seems little doubt, however, that RS CVn-type stars and cataclysmic binaries are only infrequently associated with other stars, and more work will be required to determine why this is so.

I wish to express my thanks to R. L. Walker and to other members of the staff of the USNO Flagstaff Station for providing facilities and assistance with this project.

\section{References:}

Fekel, F. C. (1981), Astrophys. J. 246, 879.

Proust, D. Ochsenbein, F., and Pettersen, B. R. (1981), Astron. Astrophys. Suppl. Ser. 44, 179.

Walker, R. L. (1979), A Catalog of Visual Binaries with One or More Variable Components, unpublished. 